\title{
Glybera and the future of gene therapy in the European Union
}

\section{Norman Miller}

A recent news article discussed the progress in gene therapy, a field that has had a number of major setbacks (Gene therapies advance towards finish line. Nature Rev. Drug Discov. 10, 719-720 (2011) $)^{1}$. The recent experience of Amsterdam Molecular Therapeutics (AMT) with its investigational gene therapy alipogene tiparvovec (also known as Glybera) provides an illustration of the challenges faced by those working in the field.

Founded in 1998, this small Dutch company developed probably the first viable manufacturing platform using adeno-associated virus (AAV)-derived vectors as the gene delivery vehicle. An important advantage of this platform is that the delivered genes are not integrated into the host chromosome, thereby avoiding potential oncogenic disruptions that have been observed with integrative vectors used to treat patients with X-linked severe combined immunodeficiency. Such a platform could potentially be applied in many monogenic disorders, and perhaps also in more common diseases.

In January 2010, AMT applied to the European Medicines Agency (EMA) for registration of the first gene therapy product based on its new technology, alipogene tiparvovec, for the correction of familial lipoprotein lipase deficiency, a rare disorder in which failure to metabolize chylomicrons leads to massive hyperlipidaemia and recurrent pancreatitis. However, the EMA's Committee for Medicinal Products for Human Use (CHMP) voted against approval in June 2011, owing to what was considered to be insufficient evidence of a persistence of effect in lowering blood lipid levels or reducing the frequency of episodes of pancreatic inflammation. This decision was maintained in October 2011 following a requested re-examination by the company, despite the fact that the EMA's Committee for Advanced Therapeutics, which advises the CHMP on decisions regarding advanced therapeutics such as gene and cell therapies, concluded that these concerns could be addressed with the additional post-marketing studies that had been offered by the company.

The product's fate will now be decided by the votes of the European Commission's Standing Committee on Medicinal Products for Human Use, which has the right to accept or reject the CHMP's recommendation. An announcement by AMT on 30 January 2012 that the European Commission's Standing Committee had postponed a decision on Glybera pending receipt of additional information from the CHMP, and an announcement on 21 February 2012 that the company has in turn received a request for more information from the CHMP, are welcome news. The Standing Committee and CHMP are to be complimented in making every effort to find the best solution. If this ultimately leads to Glybera becoming available to at least some patients with familial lipoprotein lipase deficiency while further data are being collected, it could not only provide a valuable treatment for such patients but also provide a major boost to the field of gene therapy overall.

Norman Miller is at Magdalen College, University of Oxford, Oxford OX1 4AU, UK.

doi: $10.1038 / \mathrm{nrd} 3572-\mathrm{c} 1$

Published online 10 April 2012

Mullard, A. Gene therapies advance towards finish line. Nature Rev. Drug Discov. 10, 719-720 (2011).

Competing interests statement

The author declares no competing financial interests. 